\title{
Quantum Cascade Microdisk Lasers for Mid Infrared Intra- Cavity Sensing
}

\author{
Raviv Perahia, Oskar Painter \\ Department of Applied Physics, California Institute of Technology, Pasadena, CA 91125, USA \\ e-mail:rperahia@caltech.edu \\ Virginie Moreau, Michael Bahriz, Raffaele Colombelli \\ Institut d'Electronique Fondamentale, Université Paris-Sud, Orsay 91405, France \\ A.B. Krysa ${ }^{1}$, J.W. Cockburn ${ }^{2}$, J.S. Roberts ${ }^{1}$, L.R. Wilson ${ }^{2}$ \\ 1. Natl. Ctr. for III-V Technologies, Dept. of Electronic \& Electrical Engineering, Univ. of Sheffield, UK \\ 2. Dept. of Physics and Astronomy, Univ. of Sheffield, UK
}

\begin{abstract}
The design, fabrication, and testing of surface sensitive quantum cascade microdisk lasers in the mid-infrared for intra-cavity spectroscopy and integration with microfluidic delivery is presented.

C2007 Optical Society of America

OCIS codes: (140.3070) Infrared and far-infrared lasers; (140.4780) Optical resonators
\end{abstract}

The mid-infrared region of the spectrum is rich in absorption resonances of biological molecules. Proteins, carbohydrates, and nucleic acids can be both identified and their structure probed by infrared absorption spectroscopy [1]. Quantum cascade lasers (QCLs) are electrically pumped inter-subband semiconductor lasers that operate in the mid infrared (IR) [2]. Our work focuses on the design and fabrication of highly surface-sensitive QC lasers and their integration with microfluidic chips for "intra-cavity" analyte delivery.

The integration of a microfluidic chip with the laser chip requires a unique laser design. The lasers should be planar so the microfluidic chip can be easily sealed, operate at or near room temperature for fluidic incorporation, and tune lithographically to create a multi-spectral array. We have fabricated surface plasmon (SP) guided microdisk lasers as shown in Fig 1(a). The lasers were fabricated by etching a $5 \mu \mathrm{m}$ deep, $50 \mu \mathrm{m}$ in diameter, circular trench around the contacted semiconductor region, thus forming a whispering-gallery-type resonator. The circular trench, however, was left incomplete to allow for planar electrical contacting. Fig. 1(b) and Fig. 1(c) show multimode pulsed laser operation from such devices operating at a nominal wavelength of $7.4 \mu \mathrm{m}$. In parallel a polymer microfluidic chip was fabricated using soft lithography [3] and was aligned and bonded onto a laser chip. The fluidic chip was tested by cycling dyed water and clear water through the channels to confirm fluid circulation and chip adhesion.
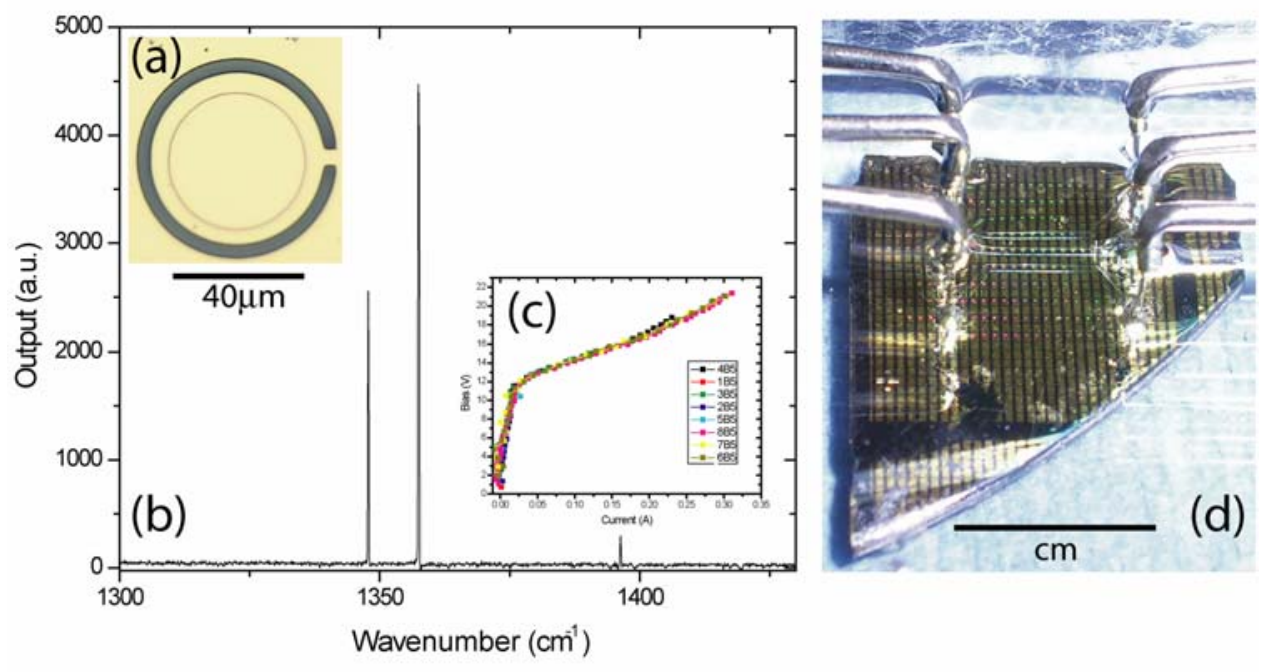

Figure 1: (a) Optical image of 50 $\mathrm{m}$ planar QC disk laser. (b) Spectral characteristics of laser in pulsed mode. Repetition rate: $84 \mathrm{kHz}$, pulse width: $50 \mathrm{~ns}$, current: $0.12 \mathrm{~A}$ (c) Current-voltage characteristics. Repetition rate: $84 \mathrm{kHz}$, pulse width: 75ns. (d) Optical image of integrated microfluidic PDMS chip with spectral array of QC micro-lasers. 


\section{CTuE5.pdf}

In order to increase the laser operating temperature and surface sensitivity of these microdisk-like lasers we have used three-dimensional, azimuthally symmetric, finite element method (FEM) simulations to optimize our designs. The vertical mode profiles are solved by modeling in cylindrical coordinates a radial slice through the structure [4]. Material absorption is taken into account by assigning each layer of the semiconductor, dielectric, and contact layer a complex index of refection. From simulations it becomes apparent that a low loss waveguide-like (WG) mode (with reasonably high active region confinement factor) is also supported in the QC laser epitaxy originally designed for SP mode operation [5]. Shown in Fig. 2(a) is a SP mode in a 25 $\mu \mathrm{m}$ diameter disk, and in Fig. 2(b) a WG mode in the same disk but with the metal contact receded by $4 \mu \mathrm{m}$ from the edge. Fig. 2(c) shows a drastic improvement in quality factor $(Q)$ as the mode transitions from SP to WG character with receding of the metal. Another contributing factor to the improved design is the removal of a top highly doped semiconductor layer $\left(\mathrm{n}^{++}\right)$usually used to form a good ohmic contact. Experimentation with different current confinement structures and other microcavity geometries in this high-quality material indicates that the top highly-doped contact layers are not necessary, and on the contrary, if removed result in much lower-loss edge-emitting SP and WG ridge lasers.

Further designs have focused on the modal properties of "microgear" resonant cavities (Fig. 2(e-f)) for modecontrol and enhanced vertical emission efficiency. In these two-dimensional simulations the effective index of the vertically guided SP and WG vertical modes were used in the metal and non metal covered regions of the disk structure. Fig. 2(e) shows the electric field intensity of a whispering-gallery-mode (WGM) which is resonant with the microgear grating. The $Q$-factor of this mode is $Q \sim 6,600$ without contacts, and is only modestly degraded $(Q \sim 5,600)$ with the addition of four $1 \mu \mathrm{m}$ wide spoke-like contacts. We have fabricated such microgear laser structures from the SP QC laser epitaxy (Fig. 2(f), and insets), and are currently studying the properties of the laser modes and their sensitivity to inserted fluids. Further modeling, fabrication, and characterization of these airguided, surface-sensitive QC microdisk and microgear devices will be presented, along with developments in the integration of microcavity QCL chips with polymer microfluidic delivery systems.
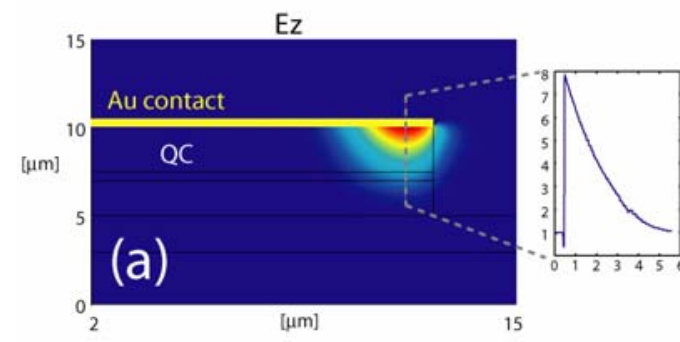

Ez
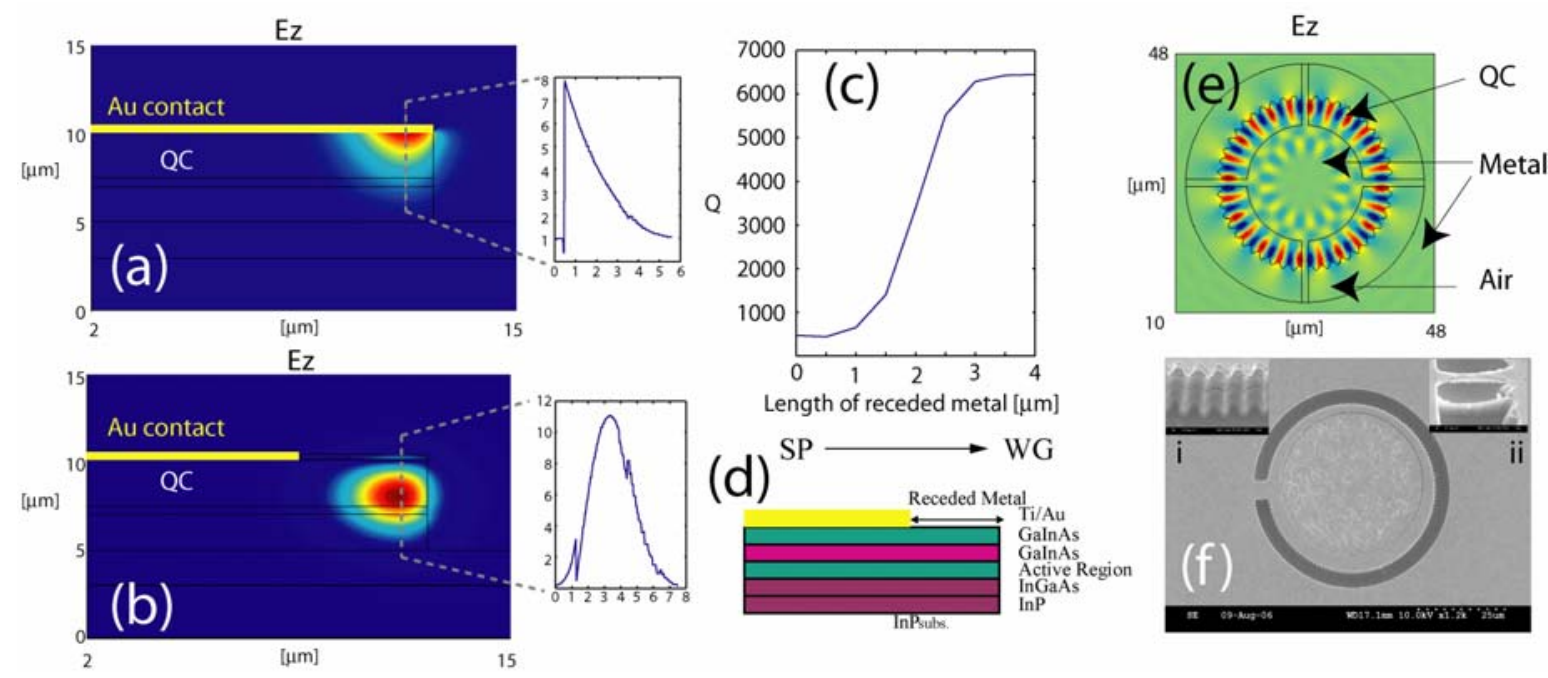

Figure 2: FEM simulation of the (a) SP mode of the fully metal covered QC microdisk, and (b) of the WG mode where a $4 \mu \mathrm{m}$ wide strip of metal is removed from the QC disk edge (c) $Q$-factor as a function of receded metal width. (d) Vertical material structure used in simulations (d) FEM simulation of a $25 \mu \mathrm{m}$ diameter microgear cavity with first order circumferential $(m=48)$ grating and four metal spoke-like contacts. (f) SEM image of fabricated 50 $\mu \mathrm{m}$ diameter microgear laser cavity with $m=124$ first order grating (inset i: zoomed in SEM of grating, inset ii: SEM of a $1 \mu \mathrm{m}$ wide air-bridged metal contact to the microdisk.

[1] L.M. Miller, G.D. Smith and G.L. Carr, "Synchrotron-based Biological Microspectroscopy: From the Mid-Infrared through the Far-Infrared Regimes," J. Bio. Phy. 29, 219 (2003).

[2] C. Gmachl, F. Capasso, D. Sivco, A.Y. Cho, "Recent progress in quantum cascade lasers and applications,” Rep. Prog. Phys. 64, 1533 (2001).

[3] M. Unger, H. Chou, T. Thorsen, A. Scherer, S. Quake, "Monolithic Microfabricated Valves and Pumps by Multilayer Soft Lithography," Science 288, 113 (2000).

[4] M. Borselli, T.J. Johnson, O. Painter, "Measuring the role of surface chemistry in silicon microphotonics," Appl. Phys. Lett. 88, 131114 (2006)

[5] M. Bahriz, V. Moreau, J. Palomo, R. Colombelli, D.A. Austin, J.W. Cockburn, L.R. Wilson, A.B. Krysa, J.S. Roberts, "Room-temperature operation of $\lambda \approx 7.5 \mu \mathrm{m}$ surface-plasmon quantum cascade lasers," Appl. Phys. Lett. 88, 181103 (2006) 